\title{
Liquid-gas Phase Transition in Strange Hadronic Matter
}

\author{
L. Yang ${ }^{1}$, W.L. Qian ${ }^{1 *}$, R.K. $\mathrm{Su}^{2,1,4 \dagger}$ and H.Q. Song ${ }^{2,3 \ddagger}$ \\ 1. Department of Physics, Fudan University,Shanghai 200433, China \\ 2. CCAST(World Laboratory), P.O.Box 8730, Beijing 100080, China \\ 3. Shanghai Institute of Applied Physics, Chinese Academy of Sciences, \\ P.O.Box 800204, Shanghai 201800, China \\ 4. Center of Theoretical Nuclear Physics, \\ National Laboratory of Heavy Ion Collisions, Lanzhou 730000, China
}

\begin{abstract}
The liquid-gas phase transition in strange hadronic matter is studied utilizing an extended Furnstahl-Serot-Tang model with nucleons and hyperons. The system is treated as of two components. The phase transition is analyzed by investigating the stability of the system and Gibbs conditions for phase equilibrium. A two-dimensional binodal surface resulted from the two-phase equilibrium, namely the phase boundary, is obtained. For each temperature ranging from $\mathrm{T}=8 \mathrm{MeV}$ to $T=12 \mathrm{MeV}$, a limit pressure on the binodal surface section is found, while a critical point is spotted for the temperature around $T=13 \mathrm{MeV}$. The Maxwell constructions are also illustrated to give a vivid description of the course of the phase transition. Moreover, the entropy per baryon and heat capacity per baryon as functions of temperature are examined. The entropy is continuous during the phase transition but the heat capacity is discontinuous, indicating that the phase transition is of second order. By these efforts, the L-G phase transition can be concluded to exist in the strange hadronic matter.
\end{abstract}

PACS numbers: 21.65.+f;11.30.Rd;21.80.+a

\footnotetext{
*wlqian@fudan.edu.cn

$\dagger$ rksu@fudan.ac.cn

$\ddagger$ songhq@sinr.ac.cn;Ref:24-430
} 


\section{INTRODUCTION}

Since the nuclear interaction is very similar to the Van der Waals potential between molecules, the possible existence of liquid-gas(L-G) phase transition in nuclear matter was predicated by theoretical studies 1, 2, 3]. This predication was supported later by intermediate-energy heavy-ion collisions [4] and high-energy proton-induced reactions [5]. The idea of an L-G phase transition in nuclear matter emerged and attracted much interest [6, 7, 8, 9, 10, 11, 12]. This interest has increased in the last decade, with the attempts by the EOS Collaboration to extract exponents of $1 \mathrm{GeV} /$ nucleon Au nuclei with a carbon target 13] and with the extraction by the ALADIN/LAND Collaboration of a caloric curve resulting from the fragmentation of the quasiprojectile formed in the collision $\mathrm{Au}+\mathrm{Au}$ at $600 \mathrm{MeV} /$ nucleon exhibiting a behavior expected for a first-order L-G phase transition [14]. Very rich information has already been extracted from exclusive experimental data during the intermediate energy heavy ion collisions, as the new generation $4 \pi$ detectors have been developed and are now operating at different accelerator facilities, such as Dubna, GANIL, GSI, LNL, LNS, MSU and Texas A-M[15].

This L-G phase transition in nuclear matter occurs at lower density than the normal nuclear density $\rho_{0}[16]$. The two phases are of cold nuclear Fermi liquid on the one hand and a nuclear gas consisting of free nucleons on the other hand. For symmetric nuclear matter, having equal number of protons and neutrons, this L-G phase transition is known of first order. However, Müller and Serot have indicated that the phase transition in asymmetric nuclear matter is of second order by Ehrenfest's definition[17]. The entropy and the volume as the first order derivatives of the chemical potentials are continuous and the heat capacity as the second order derivative is discontinuous at the point of phase transition. The phase coexistence surface of this binary system of two phases is two dimensional 17, 18, 19, 20, 21, 22, 23, 24].

Since the first hypernucleus was detected in emulsion by Danysz and Pniewski 25] in 1953, strangeness carried by s-quark has opened a new dimension for the studies in nuclear physics. With the hyperons included in, nuclear matter is extended to strange hadronic matter(SHM). Employing different models, the equation of state and the stability of the SHM have been investigated [26, 27, 28, 29, 30, 31]. However, besides the deconfinement phase transition to the quark gluon plasma(QGP), whether there exists an L-G phase transition in the SHM 
is still an open question in theoretical physics. As the interactions between hyperon and nucleon or between hyperons are also of the behavior of the Van der Waals potential, it is reasonable to guess that there will also be L-G phase transition in the SHM. It is the main purpose to study this problem.

It is believed that the descriptions of nuclear matter and finite nuclei are ultimately governed by the physics of low-energy quantum chromodynamics(QCD). Because of the nonperturbative properties of QCD, it is very difficult to establish a fundamental theory of nuclear system from QCD. One usually adopts various phenomenological models based either on hadron degree of freedom or quark degree of freedom. In our previous papers 31, 32], we suggested an effective model, constructed by introducing hyperons in the Furnstahl-SerotTang(FST) model [33, 34], to study the saturation properties and stabilities of the SHM. The details of this model can be found in ref.[31]. Here we only give a short description.

In refs. 31, 32], we extended the original FST model to include not only nucleons and $\sigma$, $\omega$ mesons, but also $\Lambda, \Xi$ hyperons. A new hyperon-hyperon interaction mediated by two additional strange mesons $\sigma^{*}$ and $\phi$, which couple only to hyperons, is introduced. The reactions $\Lambda+\Lambda \rightarrow \Xi^{-}+p, \Lambda+\Lambda \rightarrow \Xi^{0}+n$ and their reverses are considered. In this model, we did not consider the mixture of the $\Sigma$ hyperons. The reason, as was explained in ref. 31], is twofold. First, the $\Sigma$ potential in nuclear matter at the saturation density is rather uncertainly predicted, ranging from completely unbound [35] to $U_{\Sigma}=-25 \pm 5 \mathrm{MeV}[36]$. As pointed out by Balberg et al [37], systems involving $\Sigma$ 's together with nucleons or $\Lambda$ 's generally will be unstable with respect to the strong decays $\Sigma+N \rightarrow \Lambda+N$ or $\Sigma+\Lambda \rightarrow \Xi+N$. Secondly, the $\mathrm{Q}$ values for the strong transitions $\Sigma+N \rightarrow \Lambda+N, \Sigma+\Sigma \rightarrow \Lambda+\Lambda$, $\Sigma+\Lambda \rightarrow \Xi+N$ and $\Sigma+\Xi \rightarrow \Lambda+\Xi$ are about $78,156,50$ and $80 \mathrm{MeV}$, respectively [38]. To Pauli-block these processes, we need a rather high density of $\Lambda$. On the other hand, the Q value of $\Xi+N \rightarrow \Lambda+\Lambda$ is only about $28 \mathrm{MeV}$. This process can be Pauli-blocked by a relatively low $\Lambda$ density. Based on this model, the stability, the density, temperature and strangeness fraction dependence of the effective masses of nucleons and baryons, the pressure, the free energy and the equation of state are studied, and the results are reasonable.

The paper evolves from an attempt to study the L-G phase transition of SHM by employing the extended FST model. In Sec. II the extended FST model is laid out. The SHM is modelled into a two-component system ready for the discussion of phase transition. The thermodynamic quantities for this model are briefly derived out. By analyzing the 
equilibrium conditions the phase sections are addressed in Sec. III. The thermodynamical properties and the phase transition are discussed and the order of the phase transition is also examined. Sec. IV finally summarizes the main results about the existence of the L-G phase transition in the SHM.

\section{THE EXTENDED FST MODEL}

The original FST model is extended by including $\Lambda$ and $\Xi$ hyperons and two additional strange mesons $\sigma^{*}$ and $\phi$ to describe the hyperons and the interactions between them as suggested in ref. 31]. Since we are studying the unpolarized system, the $\pi$ meson has no influence on the system and is omitted. To reduce the degrees of freedom, we need some assumptions. First, we assume $\Xi^{-}$and $\Xi^{0}$ appear in equal amount. Also we have protons and neutrons in equal amount, which is similar to protons and neutrons in symmetric nuclear matter. These assumptions mean that we are looking at matter with zero isospin. But the remarkable new degree of freedom, strangeness is included. The lagrangian of the extended FST model is presented as follows:

$$
\begin{aligned}
& \mathcal{L}(x)=\bar{\psi}_{N}\left(i \gamma^{\mu} \partial_{\mu}-g_{\omega N} \gamma^{\mu} V_{\mu}-M_{N}+g_{s N} \sigma\right) \psi_{N} \\
& +\bar{\psi}_{\Lambda}\left(i \gamma^{\mu} \partial_{\mu}-g_{\omega \Lambda} \gamma^{\mu} V_{\mu}-g_{\phi \Lambda} \gamma^{\mu} \phi_{\mu}-M_{\Lambda}+g_{s \Lambda} \sigma+g_{\sigma^{*} \Lambda} \sigma^{*}\right) \psi_{\Lambda} \\
& +\bar{\psi}_{\Xi}\left(i \gamma^{\mu} \partial_{\mu}-g_{\omega \Xi} \gamma^{\mu} V_{\mu}-g_{\phi \Xi} \gamma^{\mu} \phi_{\mu}-M_{\Xi}+g_{s \Xi} \sigma+g_{\sigma^{*} \Xi} \sigma^{*}\right) \psi_{\Xi} \\
& -\frac{1}{4} G_{\mu \nu} G^{\mu \nu}+\frac{1}{2}\left(1+\eta \frac{\sigma}{S_{0}}\right) m_{\omega}^{2} V_{\mu} V^{\mu}+\frac{1}{4 !} \zeta\left(g_{\omega N}^{2} V_{\mu} V^{\mu}\right)^{2} \\
& +\frac{1}{2} \partial_{\mu} \sigma \partial^{\mu} \sigma-H_{q}\left(\frac{S^{2}}{S_{0}^{2}}\right)^{2 / d}\left[\frac{1}{2 d} \ln \left(\frac{S^{2}}{S_{0}^{2}}\right)-\frac{1}{4}\right] \\
& -\frac{1}{4} S_{\mu \nu} S^{\mu \nu}+\frac{1}{2} m_{\phi}^{2} \phi_{\mu} \phi^{\mu}+\frac{1}{2}\left(\partial_{\nu} \sigma^{*} \partial^{\nu} \sigma^{*}-m_{\sigma^{*}}^{2} \sigma^{*^{2}}\right)
\end{aligned}
$$

In mean-field approximation, the lagrangian can be written as

$$
\begin{aligned}
\mathcal{L}_{M F T}= & \bar{\psi}_{N}\left(i \gamma^{\mu} \partial_{\mu}+g_{\omega N} \gamma^{0} V_{0}-M_{N}+g_{s N} \sigma_{0}\right) \psi_{N} \\
& +\bar{\psi}_{\Lambda}\left(i \gamma^{\mu} \partial_{\mu}-g_{\omega \Lambda} \gamma^{0} V_{0}-g_{\phi \Lambda} \gamma^{0} \phi_{0}-M_{\Lambda}+g_{s \Lambda} \sigma_{0}+g_{\sigma^{*} \Lambda} \sigma_{0}^{*}\right) \psi_{\Lambda} \\
& +\bar{\psi}_{\Xi}\left(i \gamma^{\mu} \partial_{\mu}-g_{\omega \Xi} \gamma^{0} V_{0}-g_{\phi \Xi} \gamma^{0} \phi_{0}-M_{\Xi}+g_{s \Xi} \sigma_{0}+g_{\left.\sigma^{*} \Xi \sigma_{0}^{*}\right) \psi_{\Xi}}\right. \\
& +\frac{1}{2}\left(1+\eta \frac{\sigma_{0}}{S_{0}}\right) m_{\omega}^{2} V_{0}^{2}+\frac{1}{4 !} \zeta\left(g_{\omega N} V_{0}\right)^{4}+\frac{1}{2} m_{\phi}^{2} \phi_{0}^{2}-\frac{1}{2} m_{\sigma^{*}}^{2} \sigma^{*^{2}} \\
& -H_{q}\left(1-\frac{\sigma_{0}}{S_{0}}\right)^{4 / d}\left[\frac{1}{d} \ln \left(1-\frac{\sigma_{0}}{S_{0}}\right)-\frac{1}{4}\right]
\end{aligned}
$$


where the meson field operators have been replaced by their mean field values: $\phi_{0}, V_{0}, \sigma_{0}$ and $\sigma_{0}^{*} \cdot g_{i j}$ are the coupling constants of baryon $j$ to meson $i$ field. By using the standard technique of statistical mechanics, the thermodynamic potential $\Omega$ is obtained

$$
\begin{aligned}
\Omega= & V\left\{H_{g}\left[\left(1-\frac{\sigma_{0}}{S_{0}}\right)^{\frac{4}{d}}\left(\frac{1}{d} \ln \left(1-\frac{\sigma_{0}}{S_{0}}\right)-\frac{1}{4}\right)+\frac{1}{4}\right]\right. \\
& \left.-\frac{1}{2}\left(1+\eta \frac{\sigma_{0}}{S_{0}}\right) m_{\omega}^{2} V_{0}^{2}-\frac{1}{4 !} \zeta\left(g_{\omega N} V_{0}\right)^{4}-\frac{1}{2} m_{\phi}^{2} \phi_{0}^{2}+\frac{1}{2} m_{\sigma^{*}}^{2} \sigma_{0}^{*^{2}}\right\} \\
& -2 k_{B} T\left\{\sum_{i, \mathbf{k}} \ln \left[1+e^{-\beta\left(E_{i}^{*}(k)-\nu_{i}\right.}\right]+\sum_{i, \mathbf{k}} \ln \left[1+e^{-\beta\left(E_{i}^{*}(k)+\nu_{i}\right)}\right]\right\}
\end{aligned}
$$

where $\beta$ is the inverse temperature and $V$ is the volume of the system.

$$
E_{i}^{*}(k)=\sqrt{M_{i}^{* 2}+k^{2}}
$$

with the effective masses of the hyperons and nucleons

$$
\begin{gathered}
M_{i}^{*}=M_{i}-g_{s i} \sigma_{0}-g_{\sigma^{*} i} \sigma_{0}^{*} \quad(i=\Lambda, \Xi), \\
M_{i}^{*}=M_{i}-g_{s i} \sigma_{0} \quad(i=N)
\end{gathered}
$$

The mean-field values $\phi_{0}, V_{0}, \sigma_{0}$ and $\sigma_{0}^{*}$ are determined by the corresponding extreme conditions of the thermodynamic potential. For instance,the equation for $\phi$ meson is determined by

$$
\left.\frac{\partial \Omega}{\partial \phi_{0}}\right|_{\sigma_{0}, V_{0}, \sigma_{0}^{*}, \mu_{i}}=0
$$

in explicit form

$$
m_{\phi}^{2} \phi_{0}-g_{\phi \Lambda} \rho_{B \Lambda}-g_{\phi \Xi} \rho_{B \Xi}=0 .
$$

The baryon densities $\rho_{B i}$ is given by

$$
\rho_{B i}=\left\langle\psi_{i}^{+} \psi_{i}\right\rangle=\frac{g_{i}}{\pi^{2}} \int d k k^{2}\left[n_{i}(k)-\bar{n}_{i}(k)\right]
$$

where $g_{i}=4$ for $i=N$ or $\Xi, g_{i}=2$ for $i=\Lambda$. The baryon and anti-baryon distributions are, respectively, expressed as

$$
n_{i}(k)=\left\{\exp \left[\beta\left(E_{i}^{*}(k)-\nu_{i}\right)\right]+1\right\}^{-1}
$$

and

$$
\bar{n}_{i}(k)=\left\{\exp \left[\beta\left(E_{i}^{*}(k)+\nu_{i}\right)\right]+1\right\}^{-1}
$$


in which $\nu_{i}$ are related to chemical potential $\mu_{i}$ by

$$
\begin{aligned}
& \mu_{N}=\nu_{N}+g_{\omega N} V_{0}, \\
& \mu_{\Lambda}=\nu_{\Lambda}+g_{\omega \Lambda} V_{0}+g_{\phi \Lambda} \phi_{0}, \\
& \mu_{\Xi}=\nu_{\Xi}+g_{\omega \Xi} V_{0}+g_{\phi \Xi} \phi_{0} .
\end{aligned}
$$

In the system with equal number of protons and neutrons as well as equal number of $\Xi^{0}$ and $\Xi^{-}$, the chemical equilibrium condition for the reactions $\Lambda+\Lambda \rightleftharpoons n+\Xi^{0}$ and $\Lambda+\Lambda \rightleftharpoons p+\Xi^{-}$ reads

$$
2 \mu_{\Lambda}=\mu_{N}+\mu_{\Xi}
$$

Eq.(13) implies that only two components are independent among $N, \Lambda$ and $\Xi$. The system of SHM described by the extended FST model is a two-component system. The nucleons $(p$ and $n)$ and the hyperons $\left(\Lambda, \Xi^{-}\right.$and $\left.\Xi^{0}\right)$ play the role of different components, respectively. The strangeness fraction is introduced as

$$
f_{S} \equiv \frac{\rho_{B \Lambda}+2 \rho_{B \Xi}}{\rho_{B}}
$$

which plays the similar role as that of the asymmetric parameter $\alpha=\left(\rho_{n}-\rho_{p}\right) /\left(\rho_{n}+\rho_{p}\right)$ in the asymmetric nuclear matter. We can use the same method as that in ref. [17, 18, 19, 20, 21] to address the L-G phase transition.

Following the usual procedure of statistical physics, we can easily calculate the other thermodynamic quantities from thermodynamic potential $\Omega$. For example, the pressure and entropy density are calculated by formulas $p=-\Omega / V$ and $S / V=-(\partial \Omega / \partial(1 / \beta))_{V, \mu_{i}} / V=$

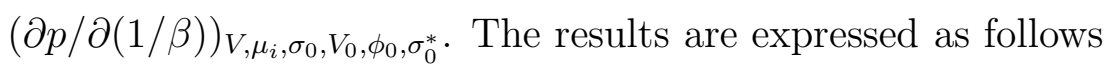

$$
\begin{gathered}
p=\sum_{i} \frac{g_{i}}{6 \pi^{2}} \int d k \frac{k^{4}}{E_{i}^{*}(k)}\left[n_{i}(k)+\bar{n}_{i}(k)\right]-H_{q}\left\{\left(1-\frac{\sigma_{0}}{S_{0}}\right)^{\frac{4}{d}}\left[\frac{1}{d} \ln \left(1-\frac{\sigma_{0}}{S_{0}}\right)-\frac{1}{4}\right]+\frac{1}{4}\right\} \\
+\frac{1}{2}\left(1+\eta \frac{\sigma_{0}}{S_{0}}\right) m_{\omega}^{2} V_{0}^{2}+\frac{1}{4 !} \zeta g_{\omega N}^{4} V_{0}^{4}+\frac{1}{2} m_{\phi}^{2} \phi_{0}^{2}-\frac{1}{2} m_{\sigma^{*}}^{2} \sigma_{0}^{*^{2}} \\
s \equiv S / V=\sum_{i} \frac{g_{i}}{6 \pi^{2}} \int d k \frac{k^{4}}{E_{i}^{*}(k)}\left\{\frac{\beta^{2}\left(E_{i}^{*}(k)-\nu_{i}\right) \exp \left[\beta\left(\left(E_{i}^{*}(k)-\nu_{i}\right)\right)\right]}{\left[\exp \left[\beta\left(\left(E_{i}^{*}(k)-\nu_{i}\right)\right)\right]+1\right]^{2}}\right. \\
\left.+\frac{\beta^{2}\left(E_{i}^{*}(k)+\nu_{i}\right) \exp \left[\beta\left(\left(E_{i}^{*}(k)+\nu_{i}\right)\right)\right]}{\left[\exp \left[\beta\left(\left(E_{i}^{*}(k)+\nu_{i}\right)\right)\right]+1\right]^{2}}\right\}
\end{gathered}
$$




\section{L-G PHASE TRANSITION}

In this section, we employ the extended FST model to investigate the L-G phase transition in the SHM at different strangeness fractions. In the calculation, the parameter set T1 given in ref. 31] is used.

As the hot and dilute SHM is only obtained on earth by the relativistic heavy ion collision, the investigations of the thermodynamic properties of the SHM are based on two basic assumptions. One is that we can apply equilibrium thermodynamics for such a small system of only few hundred constituents at the most. The other is that a thermalized uniform system is formed in heavy ion collision before the multi-fragmentation takes place[39]. Although the equilibrium analysis oversimplifies the study of the SHM, we still follow the thermodynamic approach in the reason that this can give some concrete descriptions of the phase structure of the SHM and characterize certain aspects of the evolution.

We would like to discuss the stability of SHM first. To address the stability, we consider the Helmholtz free energy density $\mathcal{F}\left(T, \rho_{i}\right)$ defined by temperature $T$ and baryon densities $\rho_{i}$. A system is stable against separation into two phases if its free energy is lower than the free energy in all two-phase configurations. This statement is formulated as

$$
\mathcal{F}\left(T, \rho_{i}\right)<(1-\lambda) \mathcal{F}\left(T, \rho_{i}^{\prime}\right)+\lambda \mathcal{F}\left(T, \rho_{i}^{\prime \prime}\right)
$$

with

$$
\rho_{i}=(1-\lambda) \rho_{i}^{\prime}+\lambda \rho_{i}^{\prime \prime}
$$

The two phases are denoted by a prime and a double prime. In asymmetric nuclear matter, we may choose $\rho_{i}$ as nuclear density $\rho$ and asymmetry parameter $\alpha$. The parameter $\lambda=$ $V^{\prime \prime} / V$ specifies the volume fraction of the phase with double prime. Eq.(18) ensures the overall baryon densities are conserved. In the SHM here, we may use variables $\rho_{B}, f_{s}$ instead of $\rho_{i}$. The above stability condition implies the following set of inequalities[17]

$$
\begin{gathered}
\rho\left(\frac{\partial p}{\partial \rho_{B}}\right)_{T, f_{s}}=\rho^{2}\left(\frac{\partial^{2} \mathcal{F}}{\partial \rho_{B}^{2}}\right)_{T, f_{s}}>0 \\
\left(\frac{\partial \mu_{N}}{\partial f_{s}}\right)_{T, p}<0 \quad \text { or } \quad\left(\frac{\partial \mu_{\Xi}}{\partial f_{s}}\right)_{T, p}>0
\end{gathered}
$$

where $p, \mu_{N}$ and $\mu_{\Xi}$ are, respectively, the pressure, chemical potentials for nucleon and $\Xi$. The first inequality Eq.(19) means that the isothermal compressibility is positive, i.e. the 
system is mechanically stable. The second condition Eq.(20) reflects the special character of the binary system. It expresses "diffusive stability" which guarantees that energy is required to change the concentration in a stable system, while holding the remaining variables ( $p$ and T) fixed.

Now we begin to discuss the region $f_{s} \leq 1.0[28]$ and show the possibility for an LG phase transition in the SHM. To be more specific, Fig.1 shows the pressure-baryon density isotherms at temperature $T=8 \mathrm{MeV}$ with different strangeness fractions $\left(f_{s}=\right.$ $0.1,0.3,0.5,0.8$ and 1.0). One can see that there is always a mechanical unstable section where condition (19) is violated for any strangeness fraction $f_{s}$ considered. This means that the system always encounters an unstable region and has to separate into two phases to maintain the mechanical stability. This phenomenon remains up to $T \simeq 13 \mathrm{MeV}$. At temperature $T=13 \mathrm{MeV}$, as is illustrated in Fig.2, there is a mechanical inflection point which satisfies the condition

$$
\left.\frac{\partial p}{\partial \rho}\right|_{f_{s}=f_{s}^{M}}=\left.\frac{\partial^{2} p}{\partial \rho^{2}}\right|_{f_{s}=f_{s}^{M}}=0
$$

with $f_{s}^{M}=0.5$ and the corresponding pressure $p=0.24 \mathrm{MeVfm}^{-3}, \rho_{B}=0.063 \mathrm{fm}^{-3}$. For $f s \geq 0.8$, it starts to decrease in high density region and mechanical unstable section will occur. However, one cannot find the solution which satisfies the Gibbs conditions in this region (See below in Fig.7). To find the temperature dependence of $f_{s}^{M}$, we also show the situation for $T=14 \mathrm{MeV}$ in Fig.3. The mechanical inflection point is around $p=0.18 \mathrm{MeV} \mathrm{fm}^{-3}$ and $\rho_{B}=0.04 \mathrm{fm}^{-3}$ on the isotherm with $f_{s}=0.1$ for $T=14 \mathrm{MeV}$. We find the $f_{s}^{M}$ decreases when the temperature increases. In Fig.4, we present the pressuredensity isotherms of fixed strangeness fraction $f_{s}=0.5$ at different temperatures. One can see again an inflection point on the curve with $T=13 \mathrm{MeV}$.

Next, we will discuss the chemical instability by showing the chemical potential isobars for nucleons and $\Xi$ against strangeness fractions $f_{s}$ at temperature $T=13 \mathrm{MeV}$ for pressure $p=0.10,0.18,0.28$ and $0.35 \mathrm{MeV} \mathrm{fm}^{-3}$, respectively, in Fig.5. There is an inflection point on the curve with pressure $p^{C}=0.28 \mathrm{MeV} \mathrm{fm}^{-3}$. This pressure is called critical pressure [17, 20], above which the system is stable but below which condition (20) is violated and the system becomes chemical unstable. The critical pressure $p^{C}$ is determined by the inflection point condition:

$$
\left(\frac{\partial \mu_{N}}{\partial f_{s}}\right)_{T, p}=\left(\frac{\partial^{2} \mu_{N}}{\partial f_{s}^{2}}\right)_{T, p}=0
$$


The result $\left(p^{C}, f_{s}^{C}\right)=\left(0.28{\mathrm{MeV} f m^{-3}}^{-3} 0.65\right)$ defines a critical point for a given temperature $T=13 \mathrm{MeV}$.

When the system separates into two phases, the two coexistent phases are governed by the Gibbs conditions, which say that for two phases (or more) the system should be in chemical, thermal and mechanical equilibrium.

$$
\begin{gathered}
\mu_{q}^{L}\left(T, \rho^{L}, f_{s}^{L}\right)=\mu_{q}^{G}\left(T, \rho^{G}, f_{s}^{G}\right),(q=N, \Xi), \\
p^{L}\left(T, \rho^{L}, f_{s}^{L}\right)=p^{G}\left(T, \rho^{G}, f_{s}^{G}\right)
\end{gathered}
$$

where the superscripts $L, G$ denote the liquid and gas phases, respectively. Here we only consider the situation of two-phase case. As to more than two phases, there is some discussion in ref.[17]. The solution $\left\{\rho^{L}, f_{s}^{L} ; \rho^{G}, f_{s}^{G}\right\}$ specifying the two separate phases in equilibrium can be easily found through a geometric approach. As an example, we present in Fig.6 the chemical-strangeness fraction isotherms at temperature $T=10 \mathrm{MeV}$ and pressure $p=0.09 \mathrm{MeV} \mathrm{fm}^{-3}$. The desired solutions form the edges of a rectangle shown by dotted lines. The phase with lower(higher) $f_{s}$ corresponding to a higher(lower) density is liquid(gas) phase. It is obvious that the strangeness fractions are different in the two phases. The collection of all such pairs $\left(p, T, f_{s}^{L}\right)$ and $\left(p, T, f_{s}^{G}\right)$ forms a binodal surface of two dimensions, which defines the phase separation boundary. The shape of the whole binodal surface is similar to that in Ref.[17, 20]. To explain clearly we show in Fig.7 a section of the binodal surface at $T=13 \mathrm{MeV}$. In Fig.7 the binodal curve is divided into two branches by zero strangeness point and critical pressure $(\mathrm{CP})$ point. The branch for larger $f_{s}$ represents the gas phase with lower density and the other for the liquid phase with higher density. In an isothermal compression, a system with zero strangeness evolves until it encounters the binodal and then remains there until the transition is completed. In an isothermal compression with $f_{s} \geq 0.8$, the L-G phase transition cannot take place because the isothermal line does not cross the binodal surface. At CP point, the liquid branch and gas branch joint together smoothly and then the two phases can no longer be distinguished by their densities. The $\mathrm{CP}$ point determines the maximum pressure in two-phase region. If the system is represented by the point in the section lower than the gas phase branch, the system is in the gas phase, whereas if the system is at the point higher than the liquid phase branch, it is in the liquid phase. Between the two branches, the system is in the mixed phase with a special proportion between liquid and gas phases. The feature of the binodal curve at temperature 
$T=13 \mathrm{MeV}$ is very similar to those in asymmetric nuclear matter [17, 20]. But for the temperature lower than $T=13 \mathrm{MeV}$, the critical points could not be attained. Instead, we can always find a limit pressure in the binodal surface. As an example, we show in Fig.8 the section of the binodal surface at the temperature $T=10 \mathrm{MeV}$, where a limit pressure $p_{\text {lim }}=0.095 \mathrm{MeV} \mathrm{fm}^{-3}$ is denoted by a horizontal dotted line. When pressure is higher than the limit pressure $p_{\text {lim }}$, the L-G phase transition cannot take place. The cut-off behavior for the binodal surface has also been found in asymmetric nuclear matter described by FST model with density-dependent $N N \rho$ coupling $g_{\rho}[18,20]$. If $g_{\rho}$ is constant, the cut-off behavior will not happen so that a critical point could always be found and no limit pressure is obtained. In this case, the L-G phase transition becomes more complicated. The limit pressure exists when $T<13 \mathrm{MeV}$ even all couplings are constant.

The Maxwell construction of the phase transition can narrate a phase transition clearly and give a concrete proof for the existence of the L-G phase transition. For a given temperature, we will expatiate the behavior of SHM under isothermal compression. At $\mathrm{T}=10 \mathrm{MeV}$, for instance, Fig.8 illustrates the situation of the isothermal compression. Assume that the system is initially in the gas phase with strangeness fraction $f_{s}=0.15$. During the compression, the phase boundary is encountered at point A. At this point, the liquid phase is about to emerge at point $\mathrm{B}$, where the strangeness fraction $f_{s}^{B}=0.05$. During the whole compression, the total strangeness fraction of the system conserves but the strangeness fractions for the liquid and gas phases are changeable. As the compression proceeds, the gas phase evolves along the gas phase branch of the binodal surface from point A to D, while the liquid phase evolves from $\mathrm{B}$ to $\mathrm{C}$ along the liquid branch. When the pressure of the system continues to increase, the system leaves the two-phase region at point $\mathrm{C}$, which has the same strangeness fraction with point A. Correspondingly, at point D on the gas branch with strangeness fraction $f_{s}=0.4$, the gas phase disappears. Since the two points A and $\mathrm{C}$ are at different branches of the binodal surface, the SHM has undergone an L-G phase transition.

To configure out the evolution of the system between point $\mathrm{A}$ and $\mathrm{C}$, we must solve the following conservation equations

$$
\begin{gathered}
\rho_{B}=(1-\lambda) \rho_{B}^{G}+\lambda \rho_{B}^{L}, \\
\rho_{B} f_{s}=(1-\lambda) \rho_{B}^{G} f_{s}^{G}+\lambda \rho_{B}^{L} f_{s}^{L},
\end{gathered}
$$


for the baryon density, strangeness fractions on the binodal surface and the proportion $\lambda$ for given total baryon density $\rho_{B}$ and strangeness fraction $f_{s}$. The proportion $\lambda$ is 0 at point $\mathrm{A}$ and 1 at point $\mathrm{C}$, and it runs through the interval $[0,1]$. The result is the generalized Maxwell construction in the binary system. The isotherms corresponding to Fig.8 are drawn in Fig.9. The system dose not evolve along the unphysical curve(the dotted line). It follows the nearly strait solid line between point $\mathrm{A}$ and $\mathrm{C}$, which is the result of the Maxwell construction. Each point on the line corresponds to the stable configuration at each intermediate density during the phase transition. Moreover, if the system is initially in the gas phase with $f_{s}=0.5$, the process of isothermal compression will make that the system begins at the gas phase, then enters a two phase region and becomes unstable at the limit pressure $p_{\text {lim }}$.

Finally, we would like to discuss the order of the L-G phase transition in the SHM. By Ehrenfest's definition, the first order phase transition is characterized by the discontinuities of the first order derivatives of the chemical potential, such as the discontinuities of entropy and volume, while the second order phase transition unfolds the discontinuous behavior for the second order derivatives of the chemical potential, such as the heat capacity. Using the entropy density of the SHM in Eq.(16), we have the entropy per baryon as

$$
s\left(T, p, f_{s}\right)=\frac{S\left(T, p, f_{s}\right)}{\rho_{B}} .
$$

The total entropy per baryon of the system is calculated by the equation:

$$
s=(1-\lambda) s^{G}+\lambda s^{L} .
$$

The entropy per baryon as a function of temperature for a matter of $f_{s}=0.50$ during the phase transition at a fixed pressure $p=0.17 \mathrm{MeV} \mathrm{fm}^{-3}$ is delineated in Fig.10. It is obvious that the entropy per baryon varies continuously during the phase transition. To make our result more transparent, we calculate the heat capacity $C_{p}$ of the SHM.

$$
\begin{aligned}
C_{p}= & T\left(\frac{\partial S}{\partial T}\right)_{p, f_{s}} \\
& =T \sum_{i} \frac{g_{i}}{6 \pi^{2}} \int d k \frac{k^{4}}{E_{i}^{*}(k)}\left[\frac{d^{2} n_{i}}{d T^{2}}+\frac{d^{2} \bar{n}_{i}}{d T^{2}}\right],
\end{aligned}
$$

where

$$
\frac{d^{2} n_{i}}{d T^{2}}=\frac{e^{\beta\left(E_{i}^{*}(k)-\nu_{i}\right)}\left[\left(E_{i}^{*}(k)-\nu_{i}\right)^{2}\left(e^{\beta\left(E_{i}^{*}(k)-\nu_{i}\right)}-1\right)-2 T\left(E_{i}^{*}(k)-\nu_{i}\right)\left(e^{\beta\left(E_{i}^{*}(k)-\nu_{i}\right)}+1\right)\right]}{T^{4}\left(e^{\beta\left(E_{i}^{*}(k)-\nu_{i}\right)}+1\right)^{3}}
$$




$$
\frac{d^{2} \bar{n}_{i}}{d T^{2}}=\frac{e^{\beta\left(E_{i}^{*}(k)+\nu_{i}\right)}\left[\left(E_{i}^{*}(k)+\nu_{i}\right)^{2}\left(e^{\beta\left(E_{i}^{*}(k)+\nu_{i}\right)}-1\right)-2 T\left(E_{i}^{*}(k)+\nu_{i}\right)\left(e^{\beta\left(E_{i}^{*}(k)+\nu_{i}\right)}+1\right)\right]}{T^{4}\left(e^{\beta\left(E_{i}^{*}(k)+\nu_{i}\right)}+1\right)^{3}}
$$

The heat capacity per baryon is expressed as $c_{p}=\frac{C_{p}}{\rho_{B}}$. In Fig.11. the specific heat capacity as a function of temperature for a fixed pressure $p=0.17 \mathrm{MeV} \mathrm{fm}^{-3}$ for $f_{s}=0.50$ is presented. A finite discontinuity of the heat capacity is observed, which demonstrates clearly that the L-G phase transition in SHM is of second order.

Till now, we can draw out a definite conclusion that an L-G phase transition do exist in the SHM and it is of second order.

\section{SUMMARY}

In this paper we have employed an extended FST model with nucleons and $\Lambda, \Xi$ hyperons to describe the thermodynamical properties of SHM. By using the reactions between hyperons and nucleons and making some assumptions, we have simplified the SHM into a two-component system, which is mathematically feasible to be discussed. When having set the strangeness fraction to be zero, the model can also obtain results, which match that of symmetric nuclear matter. Applying the model, we have investigated the L-G phase transition of the SHM. The mechanical unstable region always exists for all strangeness fractions at the temperatures lower than $T=13 \mathrm{MeV}$. Meanwhile, chemical instability is also found in chemical potential-strangeness fraction isobars at these temperatures. By using the Gibbs conditions of phase equilibrium, we have figured out the binodal surface as the phase separation boundary and have found a limit pressure at each temperature, above which there is no L-G phase transition. The limit pressure gives rise to a cut-off on the section of the binodal surface. But for temperature as high as $T=13 \mathrm{MeV}$, a critical pressure has been obtained in the binodal surface section. At the critical pressure point the liquid and gas branches joint together, resulting in a closed binodal curve. The Maxwell constructions for the phase transition have been depicted in detail. The entropy and heat capacity have also been examined and the L-G phase transition in the SHM has been determined to be of second order. 


\section{ACKNOWLEDGEMENTS}

This work is supported in part by National Natural Science Foundation of China under Nos. 10235030, 10247001, 10375013, 10347107 10047005, 10075071 by the National Basic Research Programme 2003CB716300, by the Foundation of Education Ministry of China under contract 2003246005 and CAS Knowledge Innovation Project No. KJCX2-N11.

[1] W.A. Küpper, G. Wegmann and E.R. Hilf, Ann. Phys. 88,454(1972).

[2] D.Q. Lamb et al., Phys. Rev. Lett.41,1623(1978).

[3] G. Bertsch and P. J. Siemens Phys. Lett. B126,9 (1983).

[4] M.W. Curtin, H. Toki and D.K. Scott, Phys. Lett. B123,289(1983).

[5] J.E. Finn, et al., Phys. Rev. Lett.49,1321(1982).

[6] H.R. Jaqaman, A.Z. Mekjian, and L. Zamick, Phys. Rev. C27,2782(1983);29,2067(1984).

[7] R.K. Su, S.D. Yang, and T.T.S. Kuo, Phys. Rev. C35,1539(1987).

[8] L. Satpathy, M. Mishra, and R. Nayak, Phys. Rev. C39,162(1989).

[9] J. Kapusta, Phys. Rev. C29,1735(1984).

[10] A.L. Goodman, J.I. Kapusta, and A.Z. Mekjian, Phys. Rev. C30,851(1984).

[11] D.H. Boal and A.L. Goodman, Phys. Rev. C33,1690(1986).

[12] P.J. Siemens, Nature(london)305, 410(1983).

[13] J.B. Elliott et al., Phys. Rev. C49,3185(1994); M.L. Gilkes et al., Phys. Rev. Lett. 73,1590(1994).

[14] J. Pochodzalla et al., Phys. Rev. Lett. 75,1040(1995).

[15] M.D'Agostino, M.Bruno, F.Gulminelli, R.Bougault, F.Cannata, Ph.Chomaz, F.Gramegna, N.LeNeindre, A.Moroni, G.Vannini, Proceedings of the 8th International Conference on Nucleus-Nucleus Collisions, Moscow 2003; Nucl. Phys. A734,512(2004).

[16] Takuya Furuta, Akira Ono, nucl-th/0305050

[17] H. Müller and B. D. Serot, Phys. Rev. C52, 2072 (1995).

[18] W. L. Qian, R. K. Su and P. Wang, Phys. Lett. B491,90 (2000).

[19] W. L. Qian, R. K. Su and H. Q. Song, Phys. Lett. B520,217 (2001).

[20] W. L. Qian, R. K. Su and H. Q. Song, J. Phys. G28, 379 (2002). 
[21] W. L. Qian, R. K. Su, J. Phys. G29, 1023 (2003).

[22] V. M. Kolomietz, A. I. Sanzhur, S. Shlomo and S. A. Firin, Phys. Rev. C64, 024315 (2001).

[23] V. A. Karnaukhov and et. al., Phys. Rev. C67,011601 (2003).

[24] P. Pawlowski Phys. Rev. C65,044615 (2002).

[25] M. Danysz and J. Pniewski, Phil. Mag. 44, 348 (1953).

[26] K. Ikeda, H. Bando and T. Motoba, Prog. Theor. Phys. Supplement 81, 47 (1995).

[27] J. Schaffner, C. B. Dover, A. Gal, C. Greiner, D. J. Millener and H. Stocker, Ann. Phys. 235 (1994) 35

[28] J. Schaffner-Bielich and A. Gal, Phys. Rev. C62, 034311 (2000).

[29] H. J. Schulze, M. Baldo, U. Lombardo, J. Cugnon and A. Lejeune, Phys. Rev. C57, 704 (1998).

[30] Y. Zhang and R. K. Su, Phys. Rev. C65, 035202(2002), Phys. Rev. C67, 015202(2003)

[31] L. L. Zhang, H. Q. Song, P. Wang and R. K. Su J. Phys. G26, 2301 (2000).

[32] W. L. Qian, R. K. Su and H. Q. Song, Commun. Theor. Phys. 40466 (2003).

[33] R. J. Furnstahl, Hua-Bin Tang and Brian D. Serot, Nucl. Phys. A615, 441(1997).

[34] R. J. Furnstahl, Hua-Bin Tang and Brian D. Serot, Phys. Rev. C52, 1368(1995).

[35] J. Mares, E. Triedman, A. Gal and B.K. Jennings, Nucl. Phys. A594,311(1995).

[36] C.B. Dover, D.J. Millener and A. Gal, Phys. Rep.184, 1(1989).

[37] S. Balberg, A. Gal and J. Scharffner, Progr. Theor. Phys. Suppl.117, 325(1994).

[38] V.G.J. Stoks and T.-S.H. Lee, Phys. Rev. C60, 024006-1(1999).

[39] S.J. Lee, A.Z. Mekjian, Phys. Rev. C56, 2621 (1997). 


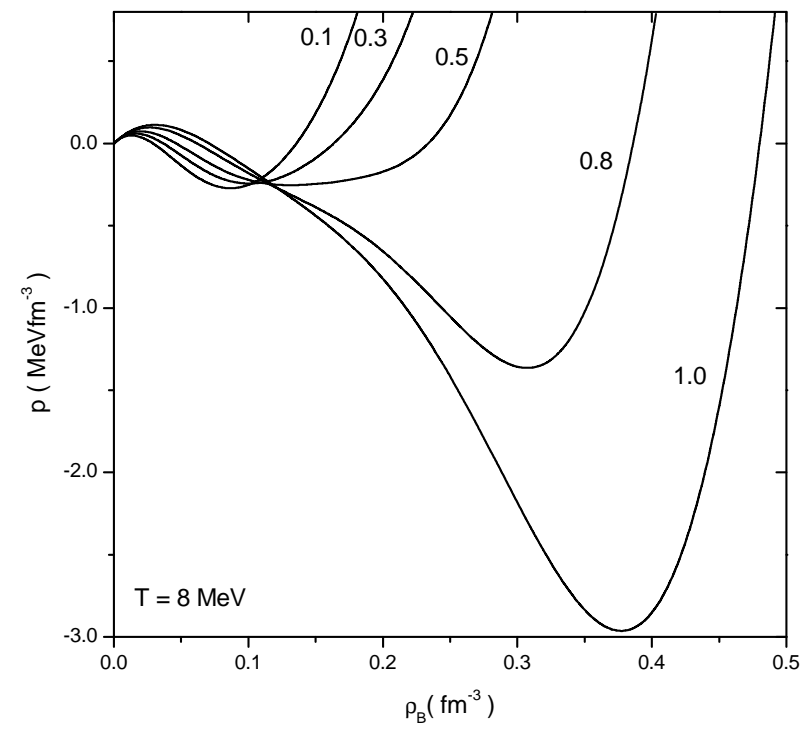

Fig.1

FIG. 1: Pressure as a function of baryon density at temperature $T=8 \mathrm{MeV}$ for various strangeness fractions $f_{s}$. 


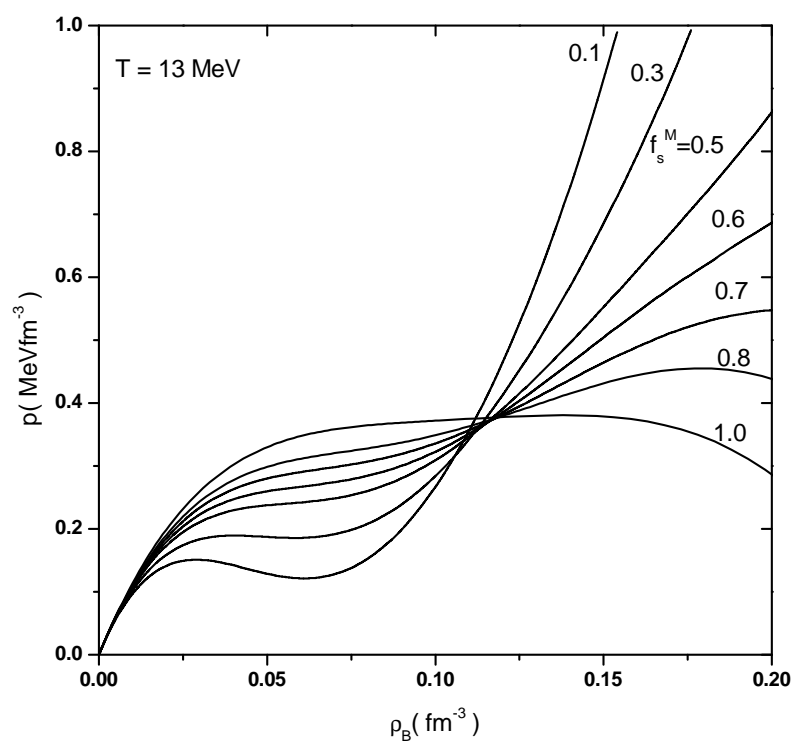

Fig.2

FIG. 2: Pressure as a function of baryon density at temperature $T=13 \mathrm{MeV}$ for various strangeness fractions $f_{s}$. The mechanical inflection point is at isotherm with $f_{s}^{M}=0.5$. 


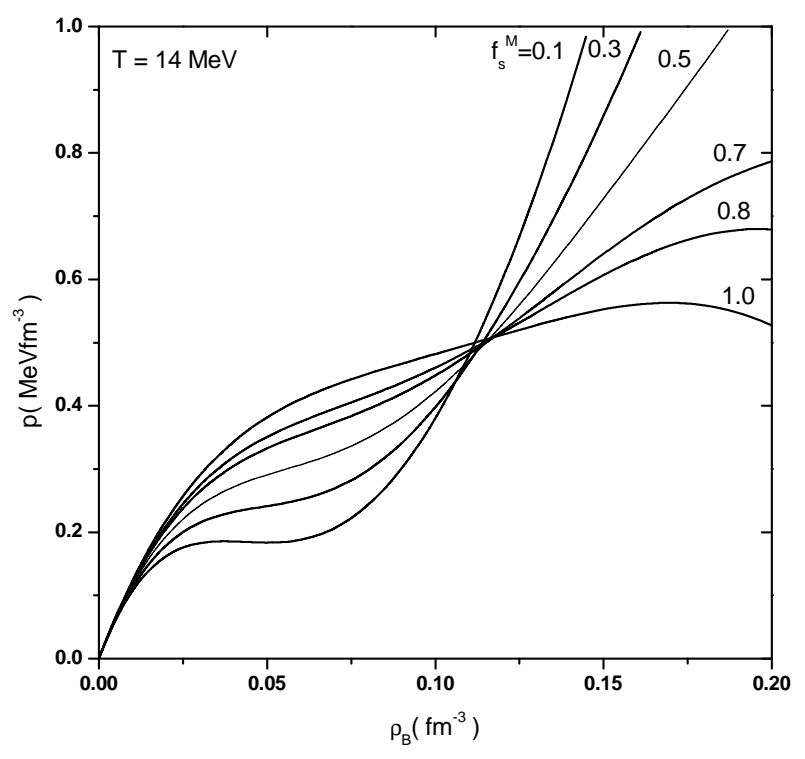

Fig.3

FIG. 3: Pressure as a function of baryon density at temperature $T=14 \mathrm{MeV}$ for various strangeness fractions $f_{s}$. A mechanical inflection point is found at isotherm with $f_{s}^{M}=0.1$. 


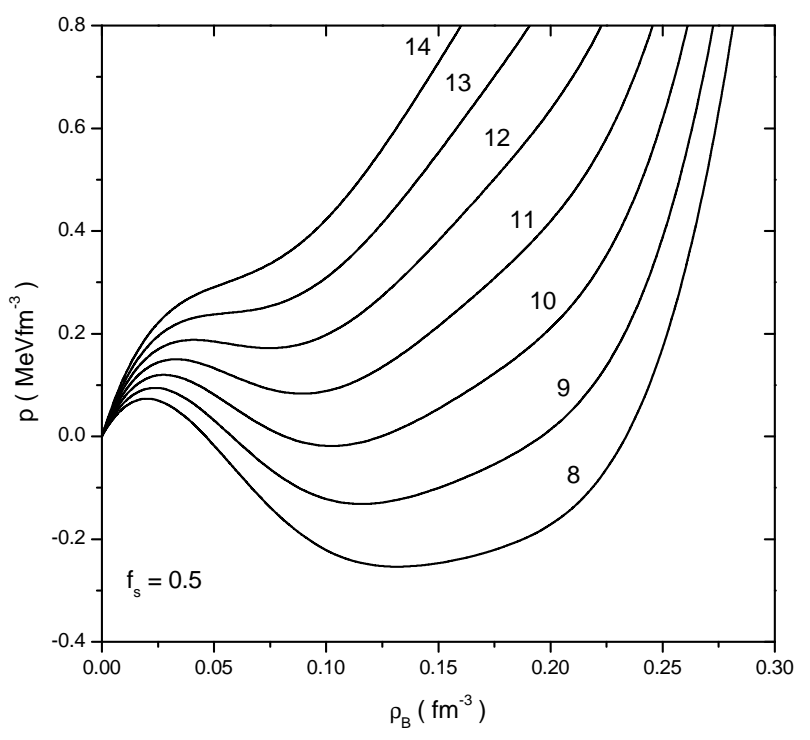

Fig.4

FIG. 4: Pressure as a function of baryon density for fixed strangeness fraction $f_{s}=0.5$ at various temperature. A mechanical inflection point is at isotherm $T=13 \mathrm{MeV}$, below which, isotherms have the mechanical unstable regions. 


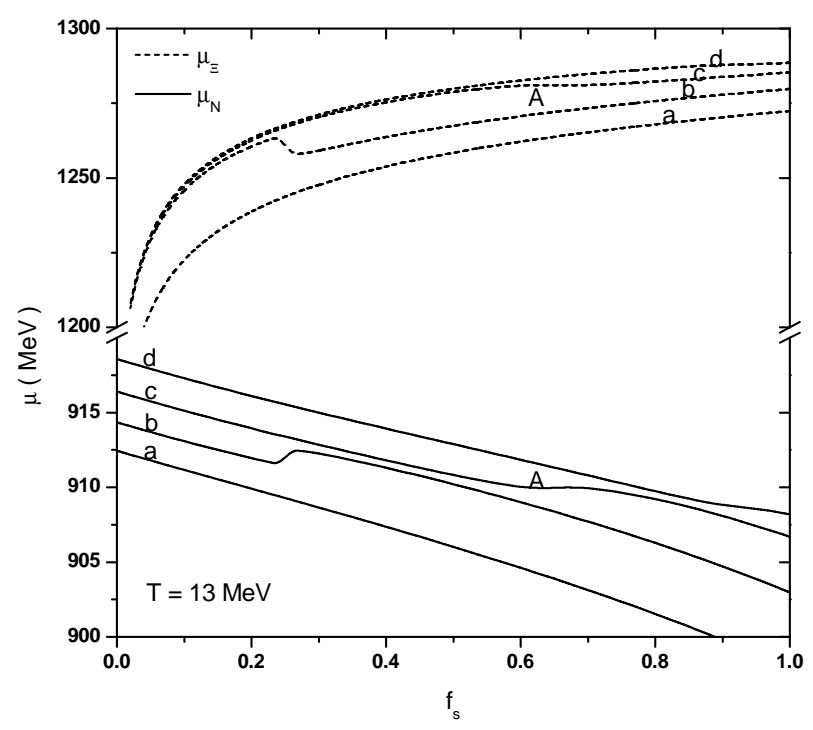

Fig.5

FIG. 5: Chemical potential isobars as a function of the strangeness fraction $f_{s}$ at fixed temperature $T=13 \mathrm{MeV}$. The curves labeled a through d have pressures $p=0.10,0.18,0.28$ and $0.35 \mathrm{MeV} \mathrm{fm}^{-3}$ respectively. Point A denotes the inflection point at the critical pressure $p^{C}=0.28 \mathrm{MeV} \mathrm{fm}^{-3}$. 


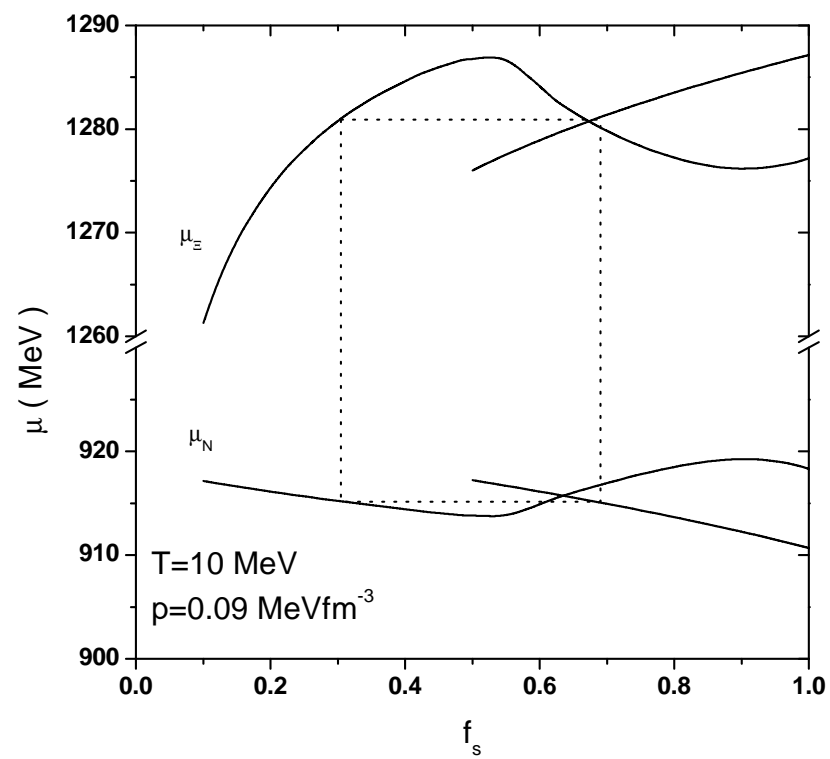

Fig.6

FIG. 6: Geometrical construction used to obtain the strangeness fraction and chemical potentials in the two coexisting phases at fixed temperature $T=10 \mathrm{MeV}$ and pressure $p=0.09 \mathrm{MeV} \mathrm{fm}^{-3}$. The two solid crossing curves for $\mu_{N}$ (or $\mu_{\Xi}$ ) are the different parts of the same continuous curve. 


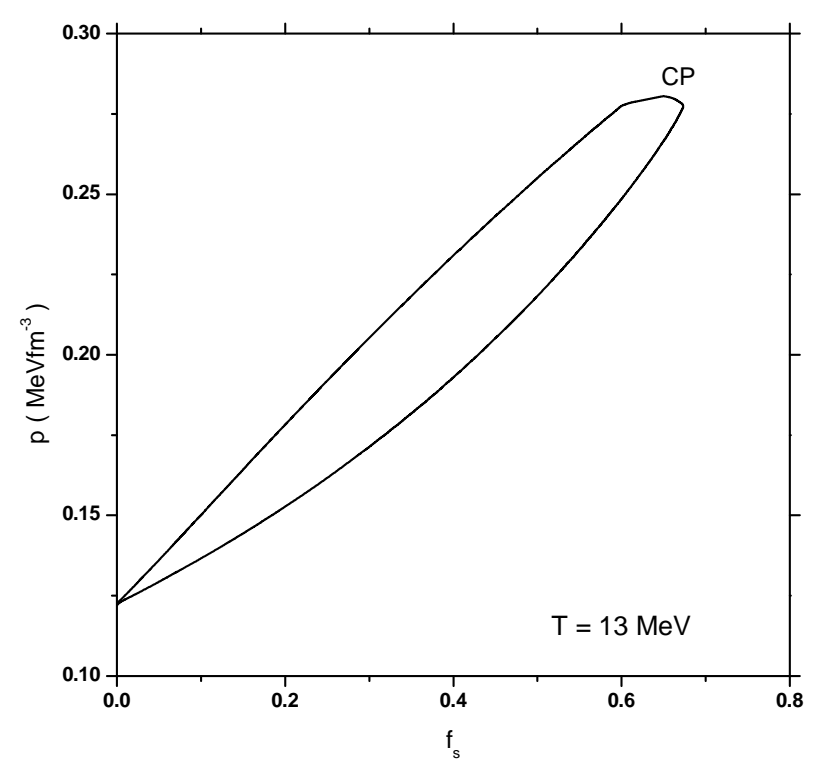

Fig.7

FIG. 7: Binodal surface at $T=13 \mathrm{MeV}$. A critical pressure $(\mathrm{CP})$ and the zero strangeness are indicated. The upper branch is for liquid phase and the lower for gas phase. Two branches joint at $\mathrm{CP}$ point. 


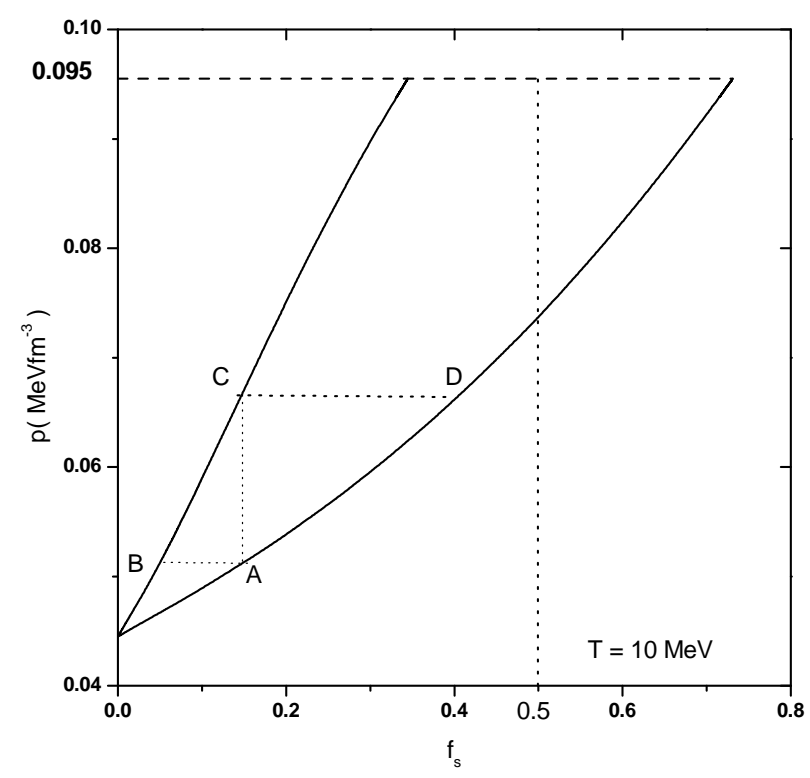

Fig.8

FIG. 8: Binodal surface at $T=10 \mathrm{MeV}$. The binodal surface is cut off at a limit pressure $p=0.095 \mathrm{MeV} \mathrm{fm}^{-3}$. The point A through $\mathrm{D}$ denote phases participating in a normal phase transition. Another phase transition for $f_{s}=0.5$ is also illustrated by the dotted line. 


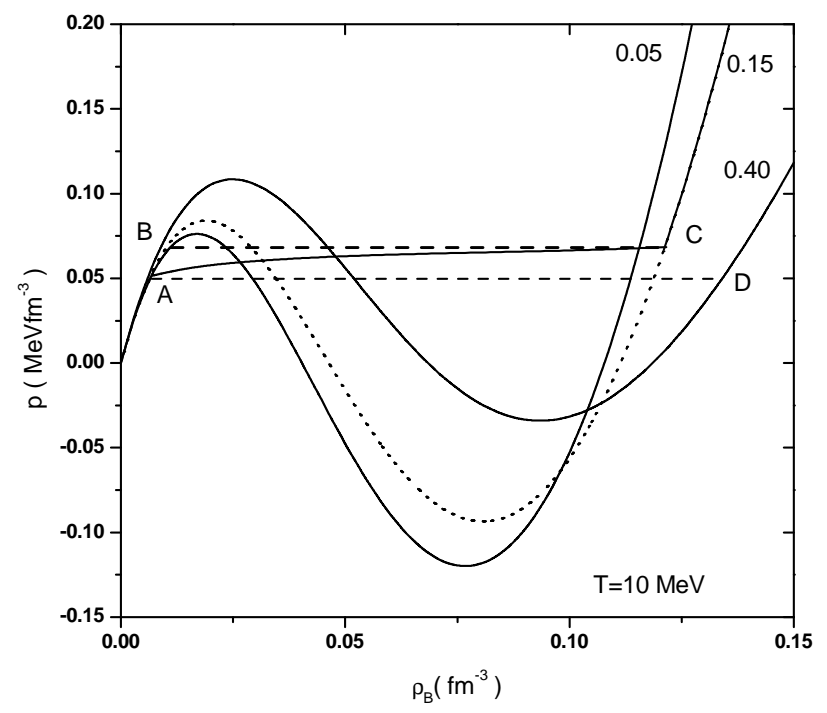

Fig.9

FIG. 9: Isotherms for a phase transition at $T=10 \mathrm{MeV}$ and the initial condition $f_{s}=0.15$. The solid line AC is built up by Maxwell construction, and the system evolves along AC. 


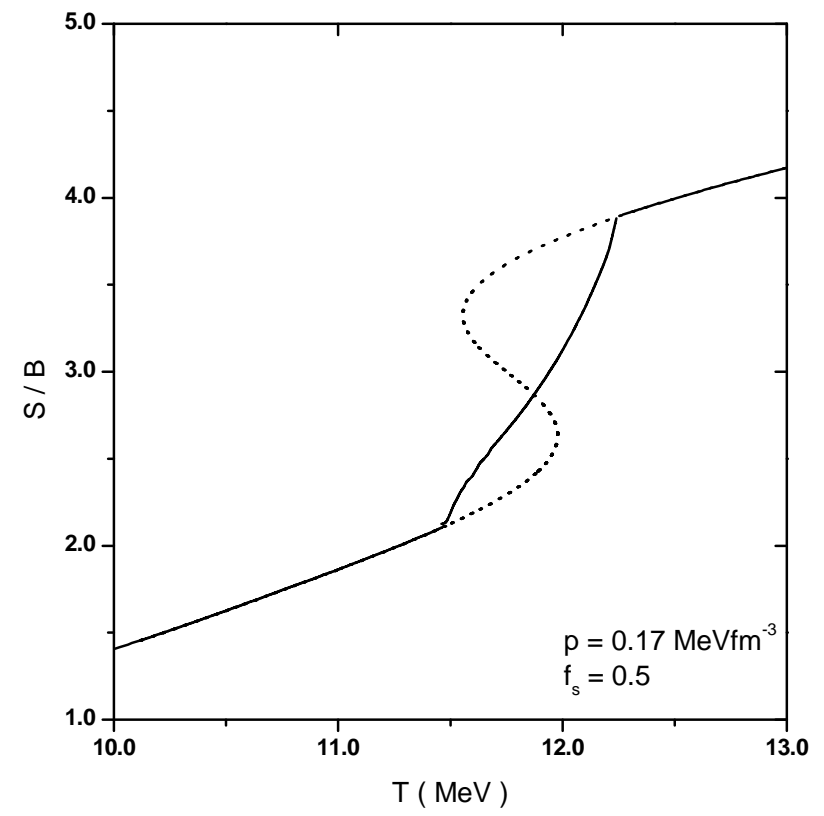

Fig.10

FIG. 10: Entropy as a function of temperature at constant pressure $p=0.17 \mathrm{MeV} \mathrm{fm}^{-3}$ for strangeness fraction $f_{s}=0.50$. The entropy evolves continuously through the phase transition. 


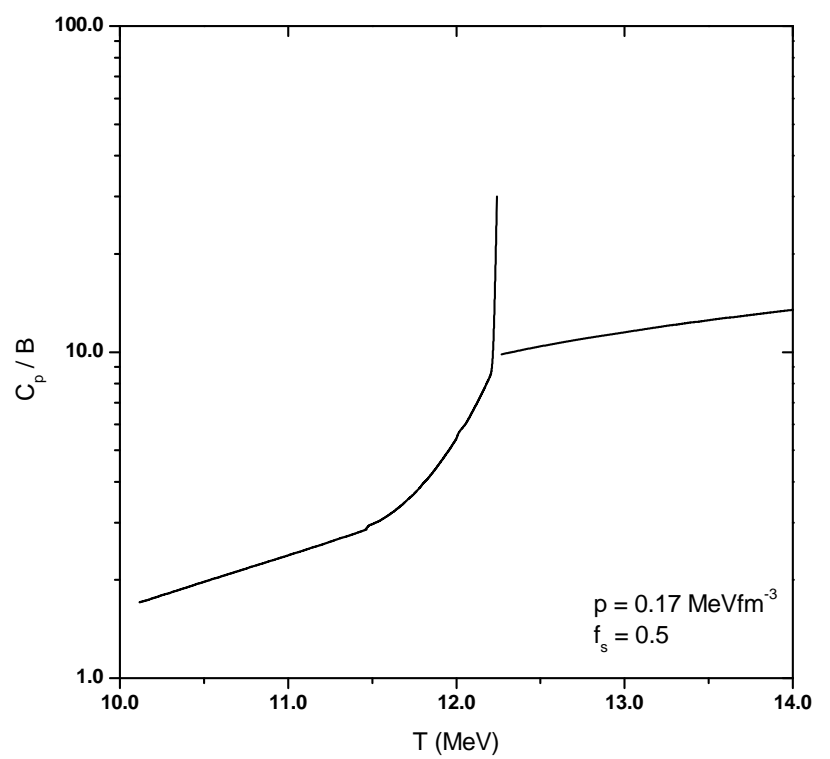

Fig.11

FIG. 11: Heat capacity as a function of temperature at fixed pressure $p=0.17 \mathrm{MeV} \mathrm{fm}^{-3}$ for strangeness fraction $f_{s}=0.50$. The discontinuity makes a strong proof for the second order phase transition. Note the logarithmic scale used on the left. 